\title{
Tecnología de membranas: obtención de proteínas de lactosuero ${ }^{1}$
}

\section{Membrane technology: obtaining whey protein}

\section{Tecnologia de membrana: obtenção de proteína de soro de leite}

\author{
J. S. Ramírez-Navas, C. A. Solís y C. A. Vélez \\ Recibido: diciembre 4 de 2017 - Aceptado: junio 15 de 2018
}

\begin{abstract}
Resumen - Las proteínas del lactosuero (LS) han adquirido gran importancia por su funcionalidad nutricional y tecnológica. En la presente revisión, se estudió el uso de la Ultrafiltración (UF) aplicada a la separación de proteínas y péptidos provenientes del LS. Se encontró que la UF es una de las mejores alternativas. Sin embargo, para lograr resultados óptimos se debe: acompañar de otras tecnologías de membranas (microfiltración, nanofiltración, electrodiálisis, diafiltración), seleccionar el tipo de membrana de UF a utilizar, y ajustar las condiciones de presión transmembranaria, $\mathrm{pH}$ y temperatura.
\end{abstract}

Palabras clave- Ultrafiltración, separación, $\alpha$-lactoalbúmina, $\beta$-lactoglobulina.

Abstract - The whey proteins have acquired great importance due to their nutritional and technological functionality. In the present review, we studied the use of Ultrafiltration (UF) applied to the separation of proteins and peptides from whey. It was found that the UF is one of the best alternatives. However, to achieve better results it is necessary

${ }^{1}$ Producto de investigación del Grupo de Investigación Ingeniería de Procesos Agroalimentarios y Biotecnológicos (GIPAB), de la Escuela de Ingeniería de Alimentos de la Universidad del Valle.

J. S. Ramírez-Navas Universidad del Valle, Cali (Colombia). email: juan.sebastian.ramirez@correounivalle.edu.co

C. A. Solís-Carvajal, Universidad del Valle, Cali (Colombia).

C. A. Vélez Pasos, Universidad del Valle, Cali (Colombia).

Como citar este artículo: Ramírez-Navas, J. S., Solís, C. A. y Vélez, C. A. Tecnología de membranas: Obtención de proteínas de lactosuero, Entre Ciencia e Ingeniería, vol. 12, no. 24, pp. 52-59, julio-diciembre, 2018. DOI: http://dx.doi.org/10.31908/19098367.3815

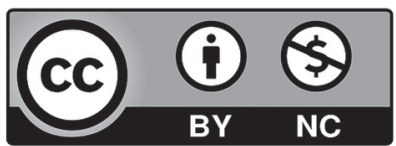

Attribution-NonCommercial 4.0 International (CC BY-NC 4.0) to: accompany other membrane technologies (microfiltration, nanofiltration, electrodialysis, diafiltration), select the type of UF membrane to be used, and adjust the conditions of transmembrane pressure, $\mathrm{pH}$ and temperature.

Keywords-Ultrafiltration, separation, $\alpha$-lactalbumin, $\beta$-lactoglobulin.

Resumo - As proteínas do soro de leite (LS) adquiriram grande importância devido à sua funcionalidade nutricional e tecnológica. Na presente revisão, estudamos o uso de Ultrafiltração (UF) aplicado à separação de proteínas e péptidos da LS. Verificou-se que a UF é uma das melhores alternativas. No entanto, para obter melhores resultados, é necessário: acompanhar de outras tecnologias de membrana (microfiltração, nanofiltração, eletrodiálise, diafiltração), selecionar o tipo de membrana UF a ser usada e ajustar as condições de pressão transmembranar, pH e temperatura.

Palavras-chave -Ultrafiltração, separação, a-lactalbumina,

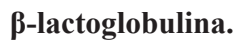

\section{INTRODUCCIÓN}

— L lactosuero (LS) es el fluido que se separa de la cuajada Een la fabricación del queso o productos similares [1]. Aproximadamente $90 \%$ del total de la leche utilizada en la elaboración del queso se elimina como LS, siendo este uno de los materiales más contaminantes que existen en la industria alimentaria. El alto poder contaminante deriva del elevado contenido de materia orgánica, y es su riqueza en lactosa la principal responsable del mismo, debido a su capacidad para actuar como sustrato de fermentación microbiana [2]. La Demanda Bioquímica de Oxígeno (DBO) del LS varía entre 20000 y $50000 \mathrm{mg}$ de $\mathrm{O}_{2} / \mathrm{L}$ [3]. En otras palabras, cerca de 250 a $300 \mathrm{~mL}$ de suero sin depurar equivale a las aguas negras producidas en un día por una persona $[4,5]$.

Por esta razón, los gobiernos de varios países exigen a las industrias de derivados lácteos una producción limpia [6], y las políticas gubernamentales son cada vez más estrictas al respecto $[7,8,9]$. Sin embargo, para Jelen [5] esta 
preocupación por la producción ecológica de los alimentos e ingredientes alimentarios puede ser una posible oportunidad para la revitalización de algunos procesos de transformación de LS, abandonados en el pasado debido a la no viabilidad económica [9].

Por otra parte, existe suficiente evidencia científica que respalda las muchas propiedades físicas, químicas, nutricionales y biológicas de las proteínas y otros componentes del LS [10]. Si se considera como alimento, su valor nutritivo es elevado. Este producto retiene cerca de $55 \%$ del total de ingredientes de la leche como la lactosa, proteínas solubles, lípidos, vitaminas y sales minerales [1, 11]. Esto ha generado un interés particular por la inclusión del LS en diversos productos alimenticios [12].

Paralelamente, el mercado es cada vez más sofisticado y receptivo a la utilidad de los componentes del LS. Esto ha hecho que actualmente sea el coproducto de la industria láctea de mayor importancia y su producción mundial crezca más del 2\% anual [10]. De sus componentes, las proteínas del LS presentan un interés particular por su composición en aminoácidos esenciales y sus excelentes propiedades funcionales [13].

El LS debe su reconceptualización de desecho a coproducto, a la tecnología de membranas (TM). Mediante la aplicación de la TM, principalmente la ultrafiltración (UF), el LS se puede concentrar para producir proteínas concentradas de suero (Whey Protein Concentrates - WPC) y aislados proteicos de suero (Whey Protein Isolates - WPI), así como fraccionar y purificar para obtener $\alpha$-lactoalbúmina ( $\alpha$-La) y $\beta$-lactoglobulina ( $\beta$-Lg) $[14,15]$. Por tanto, las proteínas de suero son una fuente considerable de ingresos para las compañías lácteas [16]. En este sentido, el objetivo de la presente revisión es exponer la ventaja de utilizar la TM en la obtención de proteínas de LS.

\section{LACTOSUERO}

La cantidad y composición del LS es muy variable ya que depende de diversos factores, por ejemplo: 1) El tipo de queso (4 L/kg de queso en quesos frescos y hasta $11,3 \mathrm{~L} / \mathrm{kg}$ de queso para quesos madurados); 2) el tratamiento térmico de la cuajada que puede disminuir el porcentaje de proteína del suero resultante; 3) la forma de coagulación (ácida o enzimática); 4) el cuajo empleado (microbiano. quimosina o mezclas quimosina/pepsina), que aporta amargor residual al LS por inactivación incompleta de las enzimas proteolíticas [17].

De acuerdo con el origen se clasifica en: LS dulce, es el líquido sobrante de la precipitación de las proteínas por hidrólisis específica de la k-caseína, por coagulación enzimática. Con $\mathrm{pH}$ próximo al de la leche inicial y sin variación de la composición mineral. El LS ácido, es el líquido sobrante obtenido después de la coagulación ácida o láctica de la caseína. Presenta un $\mathrm{pH}$ cercano a 4,5 debido a la producción de ácido láctico y alto contenido de minerales (más del 80\% de los minerales de la leche de partida) [9].

De forma general, el LS contiene los componentes solubles de la leche. En el caso del queso fresco: 4,9\% de lactosa, $0,9 \%$ de proteína, $0,6 \%$ de cenizas y $0,3 \%$ de grasas, de 6 a $7 \mathrm{~g} / \mathrm{kg}$ de proteínas [18, 19, 20, 21, 22].

\section{A. Proteínas séricas}

Las principales proteínas séricas son altamente estructuradas $\mathrm{y}$, por lo tanto, son susceptibles de ser desnaturalizadas. Están divididas en, aproximadamente: 55 $-65 \% \beta$-Lg; $15-25 \% \alpha$-La; $10-15 \%$ inmunoglobulinas; $5-6 \%$ seroalbúmina (BSA); $10-20 \%$ proteosas-peptonas; una pequeña cantidad de caseína soluble $(1-2 \%)$, proteínas menores $(<0,5 \%)$ y algunos péptidos $[18,19,20,21,22$, 23]. Sus usos son diversos, por ejemplo: $\beta$-Lg se puede usar como emulsificante, espumante y gelificante; $\alpha$-La tiene varias aplicaciones farmacéuticas, se agrega a las fórmulas infantiles, puede reemplazar la albúmina de huevo en los productos alimenticios; se utiliza como aditivo en bebidas energéticas o en productos a base de carne y pescado; la BSA (66 kDa) puede usarse como espumante y gelificante en alimentos para humanos; la lactoferrina $(86 \mathrm{kDa})$ se agrega a las fórmulas infantiles, se utiliza en cosméticos para la protección de la piel y como antibacteriano en el conservante de la carne y en la alimentación parenteral [16, 24, 25, 26, 27].

\section{a) Lactoglobulina}

La $\beta$-Lg es la proteína más abundante en el LS bovino, en el que alcanza concentraciones de 2 a $4 \mathrm{mg} / \mathrm{mL}$, representando alrededor de la mitad de las proteínas del LS. En términos de masa, constituye cerca del 50\% de las proteínas del LS. Está presente también en la leche de otras especies, como la equina y la porcina, pero no se encuentra en la leche humana. Está formada por una sola cadena de 162 aminoácidos, con un peso molecular aproximado de $36 \mathrm{kDa}$, en forma de dímero. Su secuencia se conoce desde 1976. La $\beta$-Lg en su estructura nativa, presenta capacidad de retención de agua muy moderada, del orden de $0,04 \mathrm{~g} / \mathrm{g}$ de proteína [16, 28, 29].

La $\beta$-Lg de los rumiantes (no así la de otras especies) se presenta en forma de dímeros con los monómeros unidos de forma no covalente. Estos dímeros se forman entre $\mathrm{pH} 7,5 \mathrm{y}$ $\mathrm{pH} 5,2$, el punto isoeléctrico de la $\beta$-Lg. Por encima de $\mathrm{pH}$ 7,5 y por debajo de $\mathrm{pH} 3,5$, la $\beta$-Lg se encuentra en forma de monómeros, mientras que entre $\mathrm{pH} 5,2$ y $\mathrm{pH} 3,5$ se encuentra en forma de octámeros [16, 28, 29].

La estructura terciaria de los monómeros de la $\beta$-Lg es mantenida por dos puentes disulfuro. También existe un grupo tiol libre, el correspondiente a la cisteína que ocupa el lugar 121 en la secuencia. Este tiol es muy importante en la asociación de la $\beta$ - $\mathrm{Lg}$ con otras moléculas, especialmente con la $\kappa$-caseina. Esta asociación tiene una gran influencia en la coagulación de la leche inducida por la quimosina. Los puentes disulfuro son también bastante reactivos, y dan lugar a reacciones de intercambio de sulfidrilos. La $\beta$-Lg se desnaturaliza con relativa facilidad por el calor, especialmente en ausencia de ligandos asociados [16, 28, 29].

La $\beta$-Lg es capaz de interaccionar con distintas moléculas hidrofóbicas, especialmente el retinol y los ácidos grasos. 
Esta propiedad, además de estar probablemente relacionada con su función biológica, hace que tenga buenas propiedades emulsionantes. Además, la $\beta-\mathrm{Lg}$ es la más hidrofóbica de las proteínas comunes del LS [16, 28, 29].

\section{b) Lactoalbúmina}

La $\alpha$-La es una proteína que se encuentra en la leche de casi todas las especies. Esta proteína constituye cerca del $20 \%$ de la masa de las proteínas del LS. Es la segunda proteína en concentración en el LS bovino (entre 1 y 1,5 $\mathrm{mg} / \mathrm{mL}$ ), y la más abundante en el LS humano. La $\alpha$-la es una proteína formada por una sola cadena polipeptídica de 123 componentes aminoacídicos. Contiene cuatro puentes disulfuro y es una glicoproteína que tiene cantidades variables de carbohidrato. $\mathrm{Su}$ estructura terciaria, muy compacta, globular, es mantenida por los puentes disulfuro, con una zona de hélice $\alpha$ y otra de hojas plegadas $\beta$. Es una proteína ácida con un punto isoeléctrico de alrededor de 4,8. Tiene un peso molecular aproximado de $14 \mathrm{kDa}$. Es mucho más flexible, aunque sus puentes disulfuro la mantienen en forma de un elipsoide compacto. Tiene mayor capacidad de retención de agua $(0,06 \mathrm{~g} / \mathrm{g}$ de proteína) $[29,30]$.

La $\alpha$-La tiene un ion calcio unido, que es imprescindible en el mantenimiento de su estructura y de su actividad como reguladora de la galactosiltransferasa. La eliminación del calcio produce la estructura llamada "molten globule", un estado intermedio que ha sido muy utilizado como modelo en la desnaturalización de proteínas. Este estado, con la proteína en forma "apo", es mucho menos resistente que la forma saturada con calcio a agentes desnaturalizantes, como el calentamiento [29, 31].

\section{TECNOLOGÍA DE MEMBRANAS}

La TM es un método de filtración de solutos disueltos en un fluido, cuyo fundamento es la separación por la diferencia de los pesos moleculares y el tamaño de partícula. La industria láctea ha utilizado el procesamiento de membranas desde su introducción en la industria alimentaria, a fines de la década de 1960, para clarificar, concentrar y fraccionar una variedad de productos lácteos [14]. El uso de UF y ósmosis inversa para concentrar LS fue una de las primeras aplicaciones de membranas en esta industria [15].

\section{A. Microfiltración (MF)}

La MF separa partículas con un tamaño comprendido entre 0,05 y $10 \mu \mathrm{m}$, a presiones comprendidas entre los 0,5 y 3 bar. Usualmente se usa para retener sólidos en suspensión, por lo tanto, se emplea como etapa de limpieza, de concentración o como pretratamiento [32]. La MF, en ciertas circunstancias, permite eliminar completamente bacterias y esporas (de tamaño: 0,4 a $2 \mu \mathrm{m}$ ) de la leche y derivados lácteos para extender su vida útil, convirtiéndose en una alternativa a la ultrapasteurización [33, 34]. Al aplicar la MF se puede: 1) Trabajar a temperatura más baja y, por lo tanto, evitar el sabor a cocido que se produce en el procesamiento UHT de productos lácteos de larga duración. 2) Eliminar células muertas con sus enzimas potencialmente activas que pueden causar alteraciones en las leches líquidas durante el almacenamiento [35]. 3) Aplicarse en la producción de derivados lácteos, por ejemplo, se utiliza para eliminar bacterias, esporas y grasa en la producción de WPC y WPI, logrando productos de alta calidad. Además, al aplicar MF, el tratamiento térmico del WPC y WPI se mantiene al mínimo, lo que preserva las propiedades funcionales (gelificantes, emulsionantes y espumantes) de las proteínas del LS [15].

Por otra parte, la presencia de grasa en el LS disminuye las propiedades funcionales y vida de anaquel. Para eliminar la grasa residual del LS se han desarrollado varios métodos [36]. El proceso más común aprovecha la capacidad de los fosfolípidos para agregarse en presencia de calcio, bajo un tratamiento térmico moderado durante $8 \mathrm{~min}$ a $50^{\circ} \mathrm{C}$. Este proceso se denomina precipitación termocálcica. El LS desgrasado se obtiene como permeado de MF utilizando membranas con un tamaño de poro de $0,14 \mathrm{~mm}$ [37]. El retenido, rico en fosfolípidos, puede usarse como agente emulsionante en alimentos y cosméticos. El WPC desgrasado tiene una capacidad de espuma similar a la de la clara de huevo y el mismo contenido de proteína. Sus aplicaciones pueden ser como materia prima en la producción de pasteles y helados [12].

\section{B. Ultrafiltración (UF)}

La UF es un proceso de separación a través de membranas de poros muy finos cuyo rango está entre $10 \mathrm{y}$ $1000 \AA$ [38]. La UF permite concentrar grandes moléculas y macromoléculas de peso molecular entre 1 y $200 \mathrm{kDa}$, entre las que se encuentran las del LS (ver Fig 1); por lo cual la membrana tiene un diámetro aproximado de poro de $0,01 \mu \mathrm{m}$ [39].

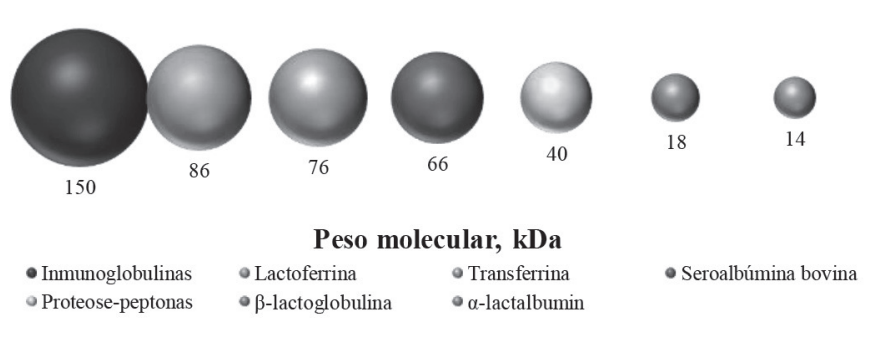

Fig. 1. Relación de pesos moleculares promedio de proteínas de lactosuero.

El límite del peso molecular de la membrana se define como el peso molecular de las proteínas globulares que son retenidas en un $90 \%$ por la membrana. La regla general (regla del dedo pulgar) es que la masa molecular debe diferir por un factor de 10 para lograr una buena separación [40]. Las moléculas que no atraviesan la membrana se conocen como material retenido o concentrado. Los materiales disueltos de bajo peso molecular pasan a través de la membrana por acción de una fuerza impulsora de presión hidrostática relativamente baja (100 - $1000 \mathrm{kPa})$; esta corriente que se forma se conoce como permeado o filtrado [41]. La UF se ha empleado con éxito en el fraccionamiento de leche, suero y proteínas [42, 43, 44]. 


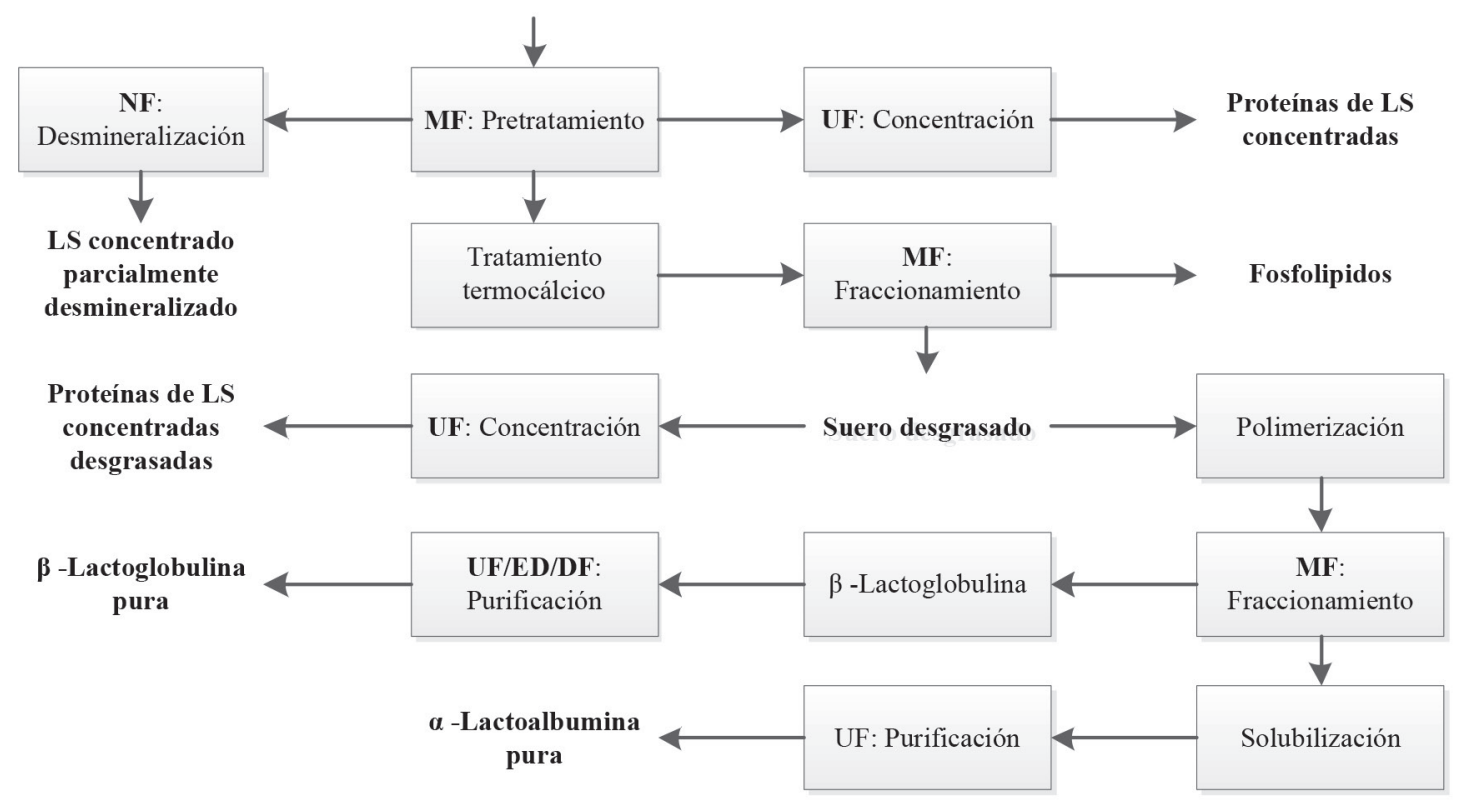

Fig. 2. Aplicación de la tecnología de membranas en la obtención de proteínas de lactosuero.

Por ejemplo, el permeado de la MF puede procesarse por UF para producir WPC de alta calidad. Estos concentrados de proteínas se pueden separar adicionalmente en lactoferrina, $\beta-\operatorname{Lg}$ y $\alpha$-Lb mediante cromatografía de intercambio iónico. Tanto la $\beta$ - $\mathrm{Lg}$ como la a-La tienen grandes mercados potenciales. $\mathrm{La} \beta-\mathrm{Lg}$ puede usarse como un agente gelificante y la a-La, que es rica en triptófano, puede usarse en la producción de péptidos con propiedades fisiológicas. Otra aplicación puede ser la producción de leche para bebés [15].

Sin embargo, debido a la complejidad del LS, es necesario usar diferentes procesos de TM y otras operaciones unitarias para producir un producto específico, como se observa en la Fig. 2., elaborada con base en Lipnizki [15], Bhattacharjee, et al. [45]. Por ejemplo, la producción de WPC con 35-85\% de proteína en el total de sólidos puede lograrse mediante la combinación de UF y Diafiltración (DF). Por otra parte, es posible mejorar la selectividad de un proceso de UF de LS ajustando el $\mathrm{pH}$ y el contenido de sal para influenciar la interacción electrostática y estérica [16].

\section{Nanofiltración (NF)}

La NF separa partículas con diámetro de poro inferior a $0,001 \mu \mathrm{m}(1 \mathrm{~nm})$. Retiene lactosa y otros componentes de gran tamaño, permitiendo permear solo iones minerales monovalentes y agua [46]. La NF permite concentrar y desmineralizar parcialmente el LS líquido. Debido a la selectividad de las membranas, la mayoría de los iones monovalentes, los ácidos orgánicos y parte de la lactosa pasarán a través de la membrana. Aproximadamente, el nivel máximo de desmineralización es del 35\% de reducción del contenido de ceniza con un factor de concentración de 3,5 a 4,0. Al aplicar un paso de DF es posible aumentar el nivel de desmineralización hasta en un $45 \%$. [15]. Un beneficio adicional de la NF es que reduce el consumo de energía y el producto de desmineralización parcial se puede secar por pulverización y usarse como aditivo alimentario o en la formulación de piensos [16].

\section{OBTENCIÓN DE $\alpha$-LA Y $\beta$-LG A PARTIR DEL LS}

La obtención de las proteínas y péptidos del LS puede lograrse mediante la separación de sus solutos disueltos por medio de una membrana de UF. En la literatura científica se presentan diversas configuraciones y tipos de membranas utilizadas, pero el empleo de metodologías diferentes conduce a que los rendimientos en el permeado y retenido varíen. Sin embargo, los factores críticos del método de UF, tales como la presión transmembranaria (PTM), el pH y la temperatura deben ser siempre valores muy similares en cualquier proceso de fraccionamiento. En la Fig. 3 se presenta un diagrama general de flujo de la UF de LS, modificado de Smith [47], Mohammadi, et al. [48]. . En esta se observa que el permeado se retira continuamente y parte del retenido es

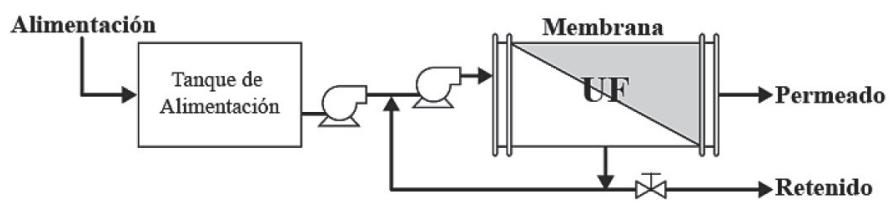

recirculado continuamente durante el proceso.

Fig. 3. Montaje de un sistema de UF (diseño parcialmente recirculado).

De acuerdo con Lipnizki [15], las proteínas purificadas de $\alpha$-La y $\beta$-Lg pueden obtenerse a partir del LS desgrasado, a un $\mathrm{pH}$ bajo $(4,0$ a 4,5$)$ y un tratamiento térmico moderado durante $30 \mathrm{~min}$ a $55^{\circ} \mathrm{C}$. Con este tratamiento la $\alpha$-La polimeriza de forma reversible atrapando a la mayoría de los lípidos residuales y otras proteínas del LS, con la excepción de la $\beta$-Lg. El fraccionamiento de la $\beta$-Lg a partir de las proteínas restantes se puede realizar mediante 
MF con un tamaño de poro de $0,2 \mathrm{~mm}$ o por centrifugación. La fase soluble resultante, rica en $\beta$ - $\mathrm{Lg}$, puede purificarse adicionalmente mediante UF junto con electrodiálisis (ED) o DF. La purificación de $\alpha$-La del retenido de MF puede lograrse mediante solubilización a $\mathrm{pH}$ neutro y posterior UF usando una membrana con un MWCO (Molecular weight cut-off) de $50 \mathrm{kDa}$.

Según Bazinet, et al. [49], se pueden fraccionar las proteínas del LS mediante membranas de electroacidificación bipolar (BMEA), sometiendo una dilución de proteína aislada de suero (WPI), de $\mathrm{pH}$ entre $6,8-7$ a un proceso de acidificación química o electroquímica; donde se obtiene un precipitado que luego de ser centrifugado durante $10 \mathrm{~min}$ a $4^{\circ} \mathrm{C}$ a $500 \times \mathrm{g}$, y liofilizado durante $24 \mathrm{~h}$, se almacena a $4{ }^{\circ} \mathrm{C}$ antes de determinar la cantidad total de proteínas. La muestra se analiza mediante la técnica de cromatografía líquida de alta eficacia (HPLC), donde se demuestra la facilidad con la que la BMEA separa las proteínas del suero, ya que con una concentración inicial de WPI al $5 \%$ se puede separar una fracción del $98 \%$ de $\beta$-Lg pura, mientras que con una WPI del $10 \%$ se puede separar una fracción del $97,3 \%$ de $\beta$-Lg y $2,7 \%$ de $\alpha$-La, para una pureza total del $98 \%$.

Muchos trabajos de investigación han centrado sus esfuerzos en encontrar la mejor relación a la cantidad de péptidos obtenidos con TM. Por ejemplo, Zydney [50] propuso el método de circulación en ambos sentidos (flujo contracorriente) con el fin de lograr extraer en una sola corrida los componentes requeridos; a esta técnica la denominó HPTTF (High-performance tangential flow filtration). Lo novedoso de este procedimiento fue que el autor no usó membranas modificadas y, por tanto, sus resultados fueron sustentados tan solo en el ajuste del $\mathrm{pH}$ al punto isoeléctrico de la proteína requerida.

Por su parte, Metsämuuronen y Nyström [51] obtuvieron mejores resultados que Zydney [50]. Los parámetros usados por ellos se resumen en la Tabla I. De esta investigación se llegó a la conclusión que la baja PTM favorece el proceso de filtración, ya que reduce la incidencia del fenómeno de polarización por concentración, y garantiza un flujo semiconstante de permeado.

TABLA I

Parámetros y Resultados de LA InVESTIGación de MetsämuUronen y NYSTRÖM

\begin{tabular}{ccc}
\hline Parámetro & Resultado & Rendimiento \\
\hline $\begin{array}{c}\text { Material de la membrana } \\
\text { hidrofilica de UF }\end{array}$ & $\mathrm{C} 030$ & \\
Temperatura & $40^{\circ} \mathrm{C}$ & $\begin{array}{c}\text { Permeado: } 79 \% \text { de } \alpha \text {-La } \\
\text { Retenido: } \beta \text {-Lg en forma de } \\
\text { pH }\end{array}$ \\
PTM & 4,3 & octámero. \\
\hline
\end{tabular}

Sin embargo, Lucas, et al. [52] demostraron que se puede separar la $\alpha$-La de la $\beta$-Lg, a partir de proteínas concentradas de suero (WPC 90\%) mediante una membrana inorgánica de UF modificada químicamente con polietilenimina (40000 $\left.\mathrm{g} \mathrm{mol}^{-1}\right)$ a $\mathrm{pH}$ neutro. Dicha membrana adquiere una carga iónica positiva que le permite interaccionar fuertemente con la $\beta$-Lg; pudiendo así separarla de la $\alpha$-La. En la Tabla II se presenta la concentración de proteínas obtenida, según Lucas, et al. [52].
TABLA II.

CONCENTRACión DE PROTEÍNAS EN LA ALIMENTACIÓN (CPL), LUEGO DEL PRE TRATAMIENTO EN POLVO (CPL 0.2) o LÍQUido (CPL 0.5)

$\begin{array}{ccc}\text { Proteína } & \text { CPL 0,2 }\left(\mathbf{g ~ L}^{-1}\right) & \text { CPL 0,5 }\left(\mathbf{g ~ L}^{-1}\right) \\ \alpha-\mathrm{La} & 1,0 \pm 0,1 & 5,0 \pm 0,2 \\ \beta-\mathrm{Lg} & 6,1 \pm 0,3 & 10,3 \pm 0,3 \\ \mathrm{BSA} & 0,20 \pm 0,05 & 0,10 \pm 0,05 \\ \mathrm{Ig} & 0,5 \pm 0,1 & 0,10 \pm 0,05\end{array}$

De igual forma, Konrad y Kleinschmidt [53], a partir de LS dulce, lograron extraer $\alpha$-La, entre el 36 y el $44 \%$ de pureza, luego de someter la corriente de alimentación a procesos previos de separación, tal como DF. Las variables de proceso usadas fueron $\mathrm{pH}$ de 6,7 , temperatura de $45^{\circ} \mathrm{C}$ y PTM de 2 bares; la investigación concluyó que el pH es el factor determinante en el aislamiento de la proteína en cuestión, resultando congruente con los reportados por Almécija, et al. [54] y Lucas, et al. [52].

Por su parte, Cheang y Zydney [55], evaluaron una forma diferente de obtener proteínas y péptidos usando membranas de UF y DF de 30 y $100 \mathrm{kDa}$, respectivamente, ordenadas en serie, con las cuales lograron obtener una buena cantidad de proteínas. Estos resultados se muestran en la Tabla III.

\begin{tabular}{|c|c|c|c|c|}
\hline & Resultai & $\begin{array}{l}\text { TABLA II } \\
\text { ABAJO D }\end{array}$ & HEANG Y & \\
\hline \multirow[t]{2}{*}{ Proteína } & \multicolumn{2}{|c|}{ CRC 100kDa, $\varepsilon=0,2(\mathrm{mS} / \mathrm{cm})$} & \multicolumn{2}{|c|}{ CRC 30kDa, $\varepsilon=1,7(\mathrm{mS} / \mathrm{cm})$} \\
\hline & So & $\Psi$ & So & $\psi$ \\
\hline$\alpha-\mathrm{La}$ & 0,67 & & 0,23 & \\
\hline$\beta-\mathrm{Lg}$ & 0,16 & 4,2 & 0,011 & 21 \\
\hline BSA & 0,009 & 74 & 0 & \\
\hline
\end{tabular}

Donde $\varepsilon$, es la conductividad; BSA, es la albumina bobina sérica; $\psi$ es la selectividad y So es el coeficiente de tamizado.

Con estos antecedentes, en algunas investigaciones posteriores combinaron técnicas de filtración para establecer cuál de estas ofrecen mejores resultados en el aprovechamiento de los péptidos. Por ejemplo, Butylina, et al. [56] trabajaron usando los métodos de UF y la NF. Concluyeron que hay mayor cantidad de péptidos en el retenido que en el permeado. En la Tabla IV se presentan los resultados obtenidos por estos autores:

$$
\text { TABLA IV }
$$

Resultados del trabajo de Butylina, ET AL.

\begin{tabular}{cccc}
\hline Componentes & Condiciones & Retentado & Permeado \\
\hline Presión $(\mathrm{PTM})$ & $0,1 \mathrm{Mpa}$ & & \\
Tipo de membrana & CM10F & & \\
Péptidos $\left(\mathrm{kg} / \mathrm{m}^{3}\right)$ & & 3,62 & 1 \\
Proteínas $\left(\mathrm{kg} / \mathrm{m}^{3}\right)$ & 3,62 & 17,3 & 0,4 \\
\hline
\end{tabular}

Asi mismo, Kanani, et al. [57] estudiaron la influencia de la forma de los poros con la cantidad de proteínas permeadas. Establecieron que los poros en forma de rejilla en el proceso tienen mayor coeficiente de tamizado y menor resistencia al flujo, que los poros en forma cilíndrica, y que entre mayor sea la distribución de estos, menor será la selectividad de la membrana. Con lo cual sienta un precedente en la elección del tipo de membrana a usar y de los parámetros más adecuados para garantizar un buen rendimiento en los procesos de obtención. 
Sin embargo, Arunkumar y Etzel [58] concluyeron que la mejor condición para fraccionar $\alpha$-La de la $\beta$ - $\mathrm{Lg}$ es a $\mathrm{pH}$ de 4,3 y conductividad de $8 \mathrm{mS} / \mathrm{cm} \quad(\psi=5,7)$, usando una membrana de $300 \mathrm{kDa}$ de carga positiva, porque el punto isoeléctrico $(5,2)$ de la $\beta$-Lg es más grande que el de la $\alpha$-La $(4,4)$. Adicionalmente, la $\beta$-Lg lleva una carga neta positiva a $\mathrm{pH}$ de 4,3 , mientras que la carga de la $\alpha$-La es cercana a cero, ello hace que preferiblemente la $\alpha$-La permee la membrana y la $\beta-\mathrm{Lg}$ sea retenida. De otro modo, observaron que la selectividad aumenta con la conductividad que se atribuye a la diferencia entre la carga neta de la $\alpha$-La y $\beta$-Lg, requiriendo mayor cantidad de sal para aumentar el coeficiente de tamizado de la $\beta$-Lg comparado con la $\alpha$-La. Sus resultados se muestran en la Tabla V.

TABLA V

CoEficiente de TAMIZADO DE A-LA Y B-LB DURANTE LA UF DE LACTOSUERO A PH DE 4.3

\begin{tabular}{cccccc}
\hline Membrana & $\mathbf{T}^{\mathbf{0}} \mathbf{C}$ & $\begin{array}{c}\text { Flujo de } \\
\text { permeado }\end{array}$ & $\begin{array}{c}\text { So } \\
\boldsymbol{\alpha} \text {-La }\end{array}$ & $\begin{array}{c}\text { So } \\
\boldsymbol{\beta} \text {-Lg }\end{array}$ & $\begin{array}{c}\text { Selectividad, } \\
(\boldsymbol{\psi})\end{array}$ \\
\hline $300 \mathrm{kDa}$ & 22 & 36 & 0,7 & 0,49 & 1,42 \\
Sin carga & 40 & 36 & 0,77 & 0,52 & 1,5 \\
& 22 & 36 & 0,35 & 0,14 & 2,5 \\
$300 \mathrm{kDa}$ & $22(\mathrm{pH} 5,2)$ & 36 & 0,61 & 0,43 & 1,4 \\
Con carga & 40 & 1,2 & 0,72 & 0,32 & 2,3 \\
\hline
\end{tabular}

\section{EJEMPLO PROCESO DE OBTENCIÓN DE LAS PROTEÍNAS}

A manera de ejemplo de un proceso de UF, se presenta el proceso de obtención de proteínas de LS realizado en la Universidad del Valle, por Murcia Muñoz [59], quien empleó una membrana de $10 \mathrm{kDa}$ con un área de filtración de $0,5 \mathrm{~m}^{2}$. Para el proceso de UF preparó $18 \mathrm{~L}$ de LS con 1500 g de LS deshidratado, obteniendo la siguiente composición:

TABLA VI

COMPOSICIÓN DEL LACTOSUERO OBTENIDO

\begin{tabular}{cccc}
\hline Componente & $\mathbf{\% p} / \mathbf{p}$ & Concentración (g/L) & \% Teórico \\
\hline Agua & 92,1 & & $92-94$ \\
Proteínas & 0,7 & 7,7 & $0,8-1$ \\
Lactosa & 6,4 & 65,8 & $4,5-6$ \\
Acidez & 0,14 & 1,4 & $0,14-0,15$ \\
Cenizas & 0,6 & 6,5 & $<0,25$ \\
Grasa & $<0,1$ & $<0,5$ & \\
\hline
\end{tabular}

En la investigación se empleó un diseño experimental central compuesto de dos factores (Q y PTM) y dos niveles por cada factor (Q: $200 \mathrm{~L} / \mathrm{h}, 300 \mathrm{~L} / \mathrm{h})$ y $(\mathrm{PTM}$ : $10 \mathrm{psi}, 20$ psi), además de tres réplicas en el punto central, realizadas de forma intercalada (una al inicio, otra en la parte central y la última al final de la experimentación), para asegurar la replicabilidad de los resultados.

El procedimiento consistió en hacer pasar la corriente a través del sistema de UF compuesto por un módulo de filtración Pellicon de Millipore que contiene un casette de membranas planas $(17,8 \mathrm{~cm}$ de ancho, $21 \mathrm{~cm}$ de largo, 2,16 cm de grosor) Biomax de polietersulfón modificado, dispuestos en paralelo y separados por pantallas de polipropileno, hasta obtener un VCR de 10, es decir, hasta que el volumen del concentrado fue una décima parte del volumen inicial del lactosuero (16,2 L de permeado y 1,8 L de concentrado), obteniendo las fracciones de permeado $\mathrm{y}$ retenido. La primera fue una solución de coloración amarillo-verdoso (debido a la presencia de vitamina $\mathrm{B}_{2}$ ), traslúcida y de apariencia y consistencia similar a la del agua, mientras que el retenido o concentrado presentó mayor viscosidad y una consistencia similar a la de la leche, pero menos traslúcido que la solución original; es en esta donde se encuentran principalmente las proteínas del LS $\alpha$-La y $\beta$-Lg, ya que son las más abundantes, tienen pesos moleculares ligeramente mayores al corte de la membrana, pero menores al criterio que garantiza una retención absoluta. En la Tabla VII se presentan los valores de concentración de lactosa y proteínas obtenidas por Murcia Muñoz [59].

TABLA VII

CONCENTRACIÓN DE LAS FRACCIONES FINALES DE PROTEÍNA Y LACTOSA OBTENIDAS MEDIANTE UF

\begin{tabular}{|c|c|c|c|c|c|}
\hline \multirow{2}{*}{$\begin{array}{c}\text { Corrida } \\
\text { Q (L/h), PTM } \\
(\text { psi })\end{array}$} & \multicolumn{2}{|c|}{ Lactosa } & \multicolumn{2}{|c|}{ Proteína } & \multirow{2}{*}{$\begin{array}{c}\% \\
\text { sobre } \\
\text { extracto seco }\end{array}$} \\
\hline & Conc. (g/L) & $\% \mathrm{Y}$ & $\begin{array}{c}\text { Conc. } \\
\text { (g/L) }\end{array}$ & $\% \mathrm{Y}$ & \\
\hline 200,10 & 79,6 & 56,7 & 15,8 & 96,7 & 19,9 \\
\hline 200,20 & 216,5 & 32,3 & 73,8 & 94,5 & 34,1 \\
\hline 300,10 & 144,4 & 26,2 & 60,9 & 94,9 & 42,1 \\
\hline 300,20 & 155,5 & 23,1 & 73,8 & 93,9 & 47,4 \\
\hline 250,15 & 203,8 & 30,9 & 72,9 & 95 & 35,8 \\
\hline 150,15 & 176,7 & 26,8 & 72,3 & 94,3 & 40,9 \\
\hline 350,15 & 142,1 & 21,4 & 72,9 & 94,3 & 51,3 \\
\hline 250,50 & 72,2 & 70 & 11,8 & 97,9 & 16,3 \\
\hline 250,25 & 135,8 & 20,4 & 71,9 & 92,9 & 53,0 \\
\hline
\end{tabular}

Como se observa, la concentración de proteínas $(\mathrm{g} / \mathrm{L})$ obtenida es superior a la encontrada en la solución inicial de LS que fue de 7,7 (g/L). No obstante, ya que los resultados anteriores fueron obtenidos con una reducción del volumen concentrado (VCR) de 10, es decir, hasta que el volumen del concentrado fue una décima parte del volumen inicial del LS (16,2 $\mathrm{L}_{\text {permeado }}$ y $1,8 \mathrm{~L}_{\text {concentrado }}$ ) y el Js (flujo de permeado) no fue un valor constante, el autor decidió ajustar el VCR a 6, con el fin de alcanzar un valor constante en el valor del flujo asumiéndolo como flujo en estado estacionario. A continuación, se muestra la ecuación que representa el VCR.

$$
V C R=\frac{V_{\text {lactosuero }}}{V_{\text {concentrado }}}
$$

En la Tabla VIII se presenta el valor de Js para cada corrida, el porcentaje de reducción del flujo desde el flux inicial hasta Js., donde las condiciones ideales de procesamiento serían aquellas que representen un valor de Js alto y un porcentaje de reducción del flujo bajo, de acuerdo con Murcia Muñoz [59]. 
TABLA VIII

CAMBIO DEL FLUJO EN ESTADO ESTACIONARIO (JS) CON LAS CONDICIONES DE PROCESO. $\mathrm{J}_{0}$ REPRESENTA EL VALOR DEL FLUJO EN $\mathrm{T}=0$.

\begin{tabular}{cccc}
\hline $\begin{array}{c}\text { Corrida } \\
\mathbf{Q}(\mathbf{L} / \mathbf{h}), \mathbf{P T M}(\mathbf{p s i})\end{array}$ & $\mathbf{J o}\left(\mathbf{L} / \mathbf{m}^{2} \mathbf{h}\right)$ & $\mathbf{J s}\left(\mathbf{L} / \mathbf{m}^{2} \mathbf{h}\right)$ & \% reducción \\
\hline 200,1 & 29,5 & 4 & 86,3 \\
200,2 & 55,4 & 13,4 & 75,7 \\
300,1 & 30,1 & 5,2 & 82,7 \\
300,2 & 57,7 & 17,5 & 69,8 \\
250,15 & 43,8 & 11,3 & 74 \\
150,15 & 43,6 & 7,9 & 81,9 \\
350,15 & 48,3 & 14,6 & 69,9 \\
250,5 & 16,7 & 1,3 & 92,1 \\
250,25 & 67,8 & 21,2 & 68,8 \\
\hline
\end{tabular}

Finalmente, con los datos anteriores ajustados a un modelo de regresión cuadrático, con $\mathrm{R}^{2}$ de $99,5 \%$, se establece que la variable con mayor efecto sobre la respuesta fue la PTM, seguido del caudal de alimentación y el efecto de las dos variables combinadas, representado en la siguiente ecuación:

$$
\begin{aligned}
J_{s}=-1,3088+0,2945 \cdot P T M-0,0133 \cdot P T M-0,0133 \cdot Q \\
+0,0002 \cdot P T M^{2}+0,0029 \cdot P T M \cdot Q+1.84 E-6 \cdot Q^{2}
\end{aligned}
$$

Con la que obtuvo que la mejor combinación presión vs velocidad de flujo a 25 Psi es $350 \mathrm{~L} / \mathrm{h}$, con un valor de Js de $28 \mathrm{~L} / \mathrm{m}^{2} \mathrm{~h}$ (flujo relativamente alto).

\section{Conclusiones}

La UF es una interesante alternativa en el proceso de separación de proteínas y péptidos del LS, componentes que presentan funcionalidad nutricional y tecnológica. Sin embargo, para lograr mejores resultados se debe: acompañar de otras TM (MF, NF, ED, DF), seleccionar el tipo de membrana de UF a utilizar, y ajustar las mejores condiciones de presión transmembranaria, $\mathrm{pH}$ y temperatura.

\section{REFERENCIAS}

[1] J. S. Ramirez-Navas, "Diseño de procesos en Industria Láctea: Transformación de lactosuero," in Investigación aplicada a la Ingeniería de Procesos, ed San Juan de Pasto, Nariño, Colombia: Editorial UNIMAR, Universidad Mariana, 2015, pp. 53-62.

[2] M. Castillo, M. J. Jordán, A. Abellán, J. Laencina, and M. B. López, "Tecnología de aprovechamiento de lactosuero," Revista Española de Lechería, vol. 74, pp. 24-30, 1996.

[3] E. Valencia Denicia and M. L. Ramírez Castillo, "La industria de la leche y la contaminación del agua," Elementos, vol. 73, pp. 27 - 31, 2009.

[4] A. E. Inda, Queso. México: Organización de los Estados Americanos, OEA, 2000.

[5] P. Jelen, "Whey processing-Utilization and products," in Encyclopedia of Dairy Sciences, H. Roginski, Ed., ed Oxford: Elsevier, 2002, pp. 2739-2745.

[6] C. A. López Ríos, A. Zuluaga Meneses, S. N. Herrera Penagos, A. A. Ruiz Colorado, and V. I. Medina de Pérez, "Production of citric acid with Aspergillus niger NRRL 2270 from milk whey," DYNA, vol. 73, pp. 39-57, 2006.

[7] A. Chacón Villalobos, "Perspectivas actuales de la proteína unicelular (SCP) en la agricultura y la industria," Agronomía Mesoamericana, vol. 15, pp. 93-106, 2004.

[8] S. T. Yang and E. M. Silva, "Novel products and new technologies for use of a familiar carbohydrate, milk lactose," Journal of Dairy Science, vol. 78, pp. 2541-2562, 1995.
[9] J. S. Ramírez-Navas, "Aprovechamiento industrial de lactosuero mediante procesos fermentativos," Publicaciones e Investigación, vol. 6, pp. 69-83, 2012.

[10] G. W. Smithers, "Whey-ing up the options - Yesterday, today and tomorrow," International Dairy Journal, vol. 48, pp. 2-14, 2015.

[11] G. d. A. Marques, J. F. B. d. São José, D. A. Silva, and E. M. M. d. Silva, "Whey protein as a substitute for wheat in the development of no added sugar cookies," LWT - Food Science and Technology, vol. 67, pp. 118-126, 4// 2016.

[12] K. Posada, D. M. Terán, and J. S. Ramírez-Navas, "Empleo de lactosuero y sus componentes en la elaboración de postres y productos de confitería," La Alimentación Latinoamericana, vol. 292, pp. 66-75, 2011.

[13] J. S. Ramírez-Navas, "Uso de la fermentación para el aprovechamiento del lactosuero," Tecnología Láctea Latinoamericana, vol. 77, pp. 5261, Sep 2013.

[14] C. Solís, C. Vélez, and J. S. Ramírez-Navas, "Tecnología de membranas: desarrollo histórico," Entre Ciencia e Ingeniería, vol. 19, pp. 89-98, 2016.

[15] F. Lipnizki, "Cross-Flow Membrane Applications in the Food Industry," in Membrane Technology: Membranes for Food Applications, Volume 3, K.-V. Peinemann, S. Pereira Nunes, and L. Giorno, Eds., ed: John Wiley \& Sons, Inc., 2010, pp. 1-24.

[16] K. Schroën, A. M. C. van Dinther, S. Bogale, M. Vollebregt, G. Brans, and R. M. Boom, "Membrane Processes for Dairy Fractionation," in Membrane Technology: Membranes for Food Applications, Volume 3, K.-V. Peinemann, S. Pereira Nunes, and L. Giorno, Eds., ed: John Wiley \& Sons, Inc., 2010, pp. 25-44.

[17] M. d. C. Jaime Valencia, "El Suero de quesería y sus posibles aplicaciones (1 parte)," Mundo Lácteo y Cárnico, vol. 3-4, pp. 4-6, 2008.

[18] M. I. González Siso, "The biotechnological utilization of cheese whey: A review," Bioresource Technology, vol. 57, pp. 1-11, 1996.

[19] M. Becerra Fernández, "Secreción de la $\beta$-galactosidasa de Kluyveromyces Lactis," Doutoramento, Universidade da Coruña, Coruña, España, 1999.

[20] R. Singh, B. S. Sooch, K. Kaur, and J. Kennedy, "Optimization of parameters for citric acid production from cheddar cheese whey using Metschnikowia pulcherrima NCIM 3108," Journal of Biological Sciences, vol. 4, pp. 700-705, 2004.

[21] J.-L. Audic, B. Chaufer, and G. Daufin, "Non-food applications of milk components and dairy co-products: A review," Le Lait, vol. 83, pp. 417-438, 2003.

[22] Ó. J. Sánchez Toro, M. C. Ortiz Buriticá, and A. L. Betancourt Garcés, "Obtención de ácido cítrico a partir de suero de leche por fermentación con Aspergillus spp," Revista Colombiana de Biotecnología, vol. VI, pp. $43-54,2004$

[23] C. Berra, "Proteína Láctea: requisito indispensable para la competitividad," presented at the IV Seminario Internacional de Competitividad en Carnes y Leches, Medellín, Colombia, 2003.

[24] M. Boland, "Whey proteins," in Handbook of Food Proteins, G. Phillips and P. Williams, Eds., ed Cornwall, UK: Woodhead Publishing Limited, 2011, pp. 30-55.

[25] A. S. Kvistgaard, J. B. Schroder, E. Jensen, A. Setarehnejad, and A. Kanekanian, "Milk Ingredients as Functional Foods," in Milk and Dairy Products as Functional Foods, A. Kanekanian, Ed., ed: John Wiley \& Sons, Ltd, 2014, pp. 198-236.

[26] K. Petrotos, E. Tsakali, P. Goulas, and A. G. D'Alessandro, "Casein and Whey Proteins in Human Health," in Milk and Dairy Products as Functional Foods, A. Kanekanian, Ed., ed: John Wiley \& Sons, Ltd, 2014, pp. 94-146.

[27] R. McCarthy, S. Mills, R. P. Ross, G. F. Fitzgerald, and C. Stanton, "Bioactive Peptides from Casein and Whey Proteins," in Milk and Dairy Products as Functional Foods, A. Kanekanian, Ed., ed: John Wiley \& Sons, Ltd, 2014, pp. 23-54.

[28] L. Sawyer, " $\beta$-Lactoglobulin," in Advanced Dairy Chemistry, Volume 1A: Proteins: Basic Aspects, P. L. H. McSweeney and P. F. Fox, Eds., 4 ed New York, USA: Springer Science+Business Media 2013, pp. 211-260.

[29] M. Calvo, Bioquímica de los alimentos. Zaragoza, España: Universidad de Zaragoza, 2000.

[30] K. Brew, " $\alpha$-Lactalbumin," in Advanced Dairy Chemistry, Volume 1A: 
Proteins: Basic Aspects, P. L. H. McSweeney and P. F. Fox, Eds., 4 ed New York, USA: Springer Science+Business Media 2013, pp. 261273.

[31] L. Pellegrino, F. Masotti, S. Cattaneo, J. Hogenboom, and I. de Noni, "Nutritional Quality of Milk Proteins," in Advanced Dairy Chemistry, Volume 1A: Proteins: Basic Aspects, P. L. H. McSweeney and P. F. Fox, Eds., 4 ed New York, USA: Springer Science+Business Media 2013, pp. 515-538.

[32] R. Ibañez Lorente, "Estudio de la Ultrafiltación de Proteínas Modelo con Membranas Cerámicas," Tesis de Doctorado, Universidad de Granada, Granada, España, 2007.

[33] L. V. Saboyainsta and J.-L. Maubois, "Current developments of microfiltration technology in the dairy industry," Le Lait, vol. 80, pp. 541-553, 2000.

[34] E. R. Griep, Y. Cheng, and C. I. Moraru, "Efficient removal of spores from skim milk using microfiltration: Spore size and surface property considerations," Journal of Animal Science, vol. 94, pp. 338-339, 2016.

[35] L. Fernández García, S. Álvarez Blanco, and F. A. Riera Rodríguez, "Microfiltration applied to dairy streams: removal of bacteria," Journal of the Science of Food and Agriculture, vol. 93, pp. 187-196, 2013.

[36] J. Fauquant, E. Vieco, and J. L. Maubois, "Clarification des lactoserums foux par agregation y thermocalcique de la matiere grasse residualle," Le Lait, vol. 65, pp. 1-20, 1985.

[37] D. Karleskind, I. Laye, F. I. Mei, and C. V. Morr, "Chemical Pretreatment and Microfiltration for Making Delipidized Whey Protein Concentrate," Journal of Food Science, vol. 60, pp. 221-226, 1995.

[38] C. Solís, C. Vélez, and J. S. Ramírez-Navas, "Tecnología de membranas: Ultrafiltración," Entre Ciencia e Ingeniería, vol. 22, pp. 26-36, 2017.

[39] M. Rosenberg, "Current and future applications for membrane processes in the dairy industry," Trends in Food Science \& Technology, vol. 6, pp. 12-19, 1// 1995.

[40] C. J. Geankoplis, Transport processes and separation process principles, 4th ed. Upper Saddle River, NJ, USA: Prentice Hall Professional Technical Reference, 2003.

[41] S. K. Sharma, S. J. Mulvaney, and S. S. H. Rizvi, Food process engineering : theory and laboratory experiments. New York, USA: Wiley-Interscience, 2000.

[42] E. D. Bastian, S. K. Collinge, and C. A. Ernstrom, "Ultrafiltration: Partitioning of Milk Constituents into Permeate and Retentate," Journal of Dairy Science, vol. 74, pp. 2423-2434, 1991.

[43] F. V. Kosikowski and R. Jimenez-Flores, "Removal of Penicillin G from Contaminated Milk by Ultrafiltration," Journal of Dairy Science, vol. 68, pp. 3224-3233, 1985.

[44] N. Rajagopalan and M. Cheryan, "Total Protein Isolate from Milk by Ultrafiltration: Factors Affecting Product Composition," Journal of Dairy Science, vol. 74, pp. 2435-2439, 1991.

[45] S. Bhattacharjee, C. Bhattacharjee, and S. Datta, "Studies on the fractionation of $\beta$-lactoglobulin from casein whey using ultrafiltration and ion-exchange membrane chromatography," Journal of Membrane Science, vol. 275, pp. 141-150, 4/20/ 2006.

[46] A. F. de Carvalho and J. L. Maubois, Engineering Aspects of Milk and Dairy Products. Boca Raton, 2010.

[47] K. Smith, "Commercial Membrane Technology," in Membrane Processing, ed: Blackwell Publishing Ltd., 2013, pp. 52-72.

[48] T. Mohammadi, A. Kohpeyma, and M. Sadrzadeh, "Mathematical modeling of flux decline in ultrafiltration," Desalination, vol. 184, pp. $367-375,11 / 1 / 2005$.

[49] L. Bazinet, D. Ippersiel, and B. Mahdavi, "Fractionation of whey proteins by bipolar membrane electroacidification," Innovative Food Science \& Emerging Technologies, vol. 5, pp. 17-25, 3// 2004.

[50] A. L. Zydney, "Protein Separations Using Membrane Filtration: New Opportunities for Whey Fractionation," International Dairy Journal, vol. 8, pp. 243-250, 3// 1998.

[51] S. Metsämuuronen and M. Nyström, "Enrichment of $\alpha$-lactalbumin from diluted whey with polymeric ultrafiltration membranes," Journal of Membrane Science, vol. 337, pp. 248-256, 7/15/ 2009.

[52] D. Lucas, M. Rabiller-Baudry, L. Millesime, B. Chaufer, and G. Daufin, "Extraction of $\alpha$-lactalbumin from whey protein concentrate with modified inorganic membranes," Journal of Membrane Science, vol. 148, pp. 1-12, 1998.

[53] G. Konrad and T. Kleinschmidt, "A new method for isolation of native $\alpha$-lactalbumin from sweet whey," International Dairy Journal, vol. 18, pp. 47-54, 2008.

[54] M. C. Almécija, R. Ibáñez, A. Guadix, and E. M. Guadix, "Effect of $\mathrm{pH}$ on the fractionation of whey proteins with a ceramic ultrafiltration membrane," Journal of Membrane Science, vol. 288, pp. 28-35, 2007.

[55] B. Cheang and A. L. Zydney, "A two-stage ultrafiltration process for fractionation of whey protein isolate," Journal of Membrane Science, vol. 231, pp. 159-167, 3/1/ 2004.

[56] S. Butylina, S. Luque, and M. Nyström, "Fractionation of whey-derived peptides using a combination of ultrafiltration and nanofiltration," Journal of Membrane Science, vol. 280, pp. 418-426, 9/1/ 2006.

[57] D. M. Kanani, W. H. Fissell, S. Roy, A. Dubnisheva, A. Fleischman, and A. L. Zydney, "Permeability-selectivity analysis for ultrafiltration: Effect of pore geometry," Journal of Membrane Science, vol. 349, pp. 405-410, 3/1/ 2010.

[58] A. Arunkumar and M. R. Etzel, "Fractionation of $\alpha$-lactalbumin from $\beta$-lactoglobulin using positively charged tangential flow ultrafiltration membranes," Separation and Purification Technology, vol. 105, pp. $121-128,2 / 5 / 2013$.

[59] F. Murcia Muñoz, "Evaluación técnica de la concentración de proteínas de lactosuero por ultrafiltración " (Recurso electrónico), Escuela de Ingeniería Química, Universidad del Valle, Cali, Colombia, 2006.

Carlos Andrés Solís-Carvajal, es Ingeniero de Alimentos de la Universidad del Valle (Colombia). https://orcid.org/0000-0002-0032-5839

Carlos Antonio Vélez Pasos, es Ingeniero Agrícola de la Universidad Nacional de Colombia - Sede Medellín. Master en Ingeniería Agrícola y Doctor en Ingeniería de Alimentos de la Universidad de Estadual de Campinas (Brasil). Es investigador del Grupo de Investigación Ingeniería de Procesos Agroalimentarios y Biotecnológicos (GIPAB). Actualmente es Director de la Escuela de Ingeniería de Alimentos de la Universidad del Valle (Colombia). ORCID: https://orcid.org/0000-0001-9974-0639

Juan Sebastián Ramírez-Navas es Ingeniero Químico de la Universidad Central del Ecuador. Doctor en Ingeniería (énfasis en Ingeniería de Alimentos) de la Universidad del Valle (Colombia). Es investigador del Grupo de Investigación Ingeniería de Procesos Agroalimentarios y Biotecnológicos (GIPAB). Actualmente es Profesor Asistente (DOMT) en la Escuela de Ingeniería de Alimentos de la Universidad del Valle (Colombia). ORCID: https://orcid.org/0000-0002-6731-2784 